\title{
NECESSARY AND SUFFICIENT CONDITIONS FOR THE OSCILLATION OF DELAY DIFFERENTIAL EQUATION WITH A PIECEWISE CONSTANT ARGUMENT
}

\author{
H.A. AGWO \\ Department of Mathematics \\ Faculty of Education \\ Ain Shams University \\ Roxy, Cairo , Egypt.
}

(Received August 13, 1996 and in revised form March 18, 1997)

ABSTRACT: The characteristic equation for an equation with continuous and piecewise constant argument in the form

$$
\dot{x}(t)+p x(t-\tau)+q x([t-k])=0 \text { where } \mathrm{p}, \mathrm{q} \in \mathfrak{R}, \tau \in \Re^{+} \text {and } k \in \mathcal{K} .
$$

is presented, which when $q=0$ reduces to

$$
f(\lambda)=\lambda+e^{-\lambda \tau}=0
$$

and when $p=0$ reduces to

$$
\lambda-1+q \lambda^{-k}=0 .
$$

Also, the necessary and sufficient conditions for oscillation are obtained.

KEY WORDS: Oscillations, Delay differential equations.

1991 AMS SUBJECT CLASSIFICATION CODES: 34k15 ; 39 A10.

\section{INTRODUCTION}

The study of equations with piecewise constant argument was originated by the work of Wiener and his collaborators. See $[1,2,3,4,5$ and 6$]$ and the references cited therein . In addition to its own interest this area has stimulated much activity in the study of delay difference equations.

As usual, a solution $x(\mathrm{t})$ is called oscillatory if it has arbitrarily large zeros .Otherwise, the solution is called nonoscillatory.An equation is called oscillatory if all its solutions are oscillatory.

Let [.] denote the greatest-integer function, $\aleph$ the set of non-negative integers and $\mathfrak{R}$ the set of real numbers.

Consider

$$
\dot{x}(t)+p x(t-\tau)+q x([t-k])=0
$$

where $\mathrm{p}, \mathrm{q} \in \mathfrak{R}, \tau \in \mathfrak{R}^{+}$and $k \in \mathcal{N}$. 
By a solution of Eqn.(1.1), we mean a function $x$ which is defined on the set $\{-\mathrm{k}, \ldots,-1,0\} \cup$ $[-\tau, \infty)$ and satisfies the following properties:

(a) $x$ is continuous on $[-\tau, \infty)$.

(b) the derivative $\dot{x}$ exists at each point $t \in(0, \infty)$ with the possible exception of the points $t \in \mathfrak{K}$, where one side derivatives exist.

(c) Eqn.(1.1) is satisfied on each interval $[n, n+1)$ for $n \in \aleph$.

Let $\phi \in C([-\tau, 0], \Re)$ and $a_{-k}, \ldots, a_{-1}, a_{0}$ be given real numbers such that

$$
a_{-j}=\phi(-j) \text { for } j \leq \tau, j=0,1,2, \ldots, k,
$$

then one can show that Eqn. (1.1) has a unique solution satisfying the initial conditions

$$
\begin{array}{ll}
x(t)=\phi(t) & -\tau \leq t \leq 0 \\
x(-j)=a_{-j} & j=0,1, \ldots, k .
\end{array}
$$

When $q=0$, Eqn. (1.1) reduces to

$$
\dot{u}(t)+p u(t-\tau)=0
$$

which is oscillatory if and only if its characteristic equation

$$
f(\lambda)=\lambda+e^{-\lambda \tau}=0
$$

has no real roots, or equivalently, to

$$
p \tau>\frac{1}{e} .
$$

On the other hand, when $p=0$, Eqn.(1.1) reduces to

$$
\dot{v}(t)+q v([t-k])=0
$$

which is oscillatory if and only if the following equation

$$
\lambda-1+q \lambda^{-k}=0
$$

has no positive real roots, or equivalently,

$$
\begin{array}{ll}
q>\frac{k^{k}}{(k+1)^{k+1}} & , k \geq 1 \\
q \geq 1 & , k=0
\end{array}
$$

An open question arises ( see [4] ,p. 223) for obtaining a characteristic equation for equation (1.1) which reduces to Eqn.(1.5) when $q=0$ and reduces to Eqn. (1.8) when $p=0$ and also obtaining the necessary and sufficient conditions for oscillation of all solutions of

$$
\dot{x}(t)+p x(t-1)+q x([t-1])=0
$$

\section{THE MAIN RESULTS}

In the following, a characteristic equation associated with equation (1.1) will be presented in Theorem 2.1. Also the necessary and sufficient conditions for oscillation are obtained through Theorems

\section{2 and 2.3 .}

THEOREM 2.1. The characteristic equation associated with equation (1.1) is

$$
f(\lambda)=\lambda-1+\frac{p \lambda^{-\tau}}{\ln \lambda}(\lambda-1)+q \lambda^{-k}=0
$$


which reduces to Eqn. (1.5) when $q=0$ and reduces to Eqn. (1.8) when $p=0$.

PROOF: Consider Eqn.(1.1) and assume that the initial conditions (1.3a) and (!.3b) are satisfied .For $t \in[n, n+1)$, we have $[t-k]=n-k$ and one can write

$$
\begin{array}{ll}
\dot{x}(t)+p x(t-\tau)+q a_{n-k}=0 & , t \in[n, n+1) \\
x(n)=a_{n} & , n \in \aleph
\end{array}
$$

Integrating (2.2a) from $n$ to $t$, we get

$$
x(t)-a_{n}+p \int_{n}^{t} x(s-\tau) d s+q a_{n-k}(t-n)=0 .
$$

By using the continuity of $\mathrm{x}(\mathrm{t})$ as $t \rightarrow n+1$, we find

$$
a_{n+1}-a_{n}+p \int_{n}^{n+1} x(s-\tau) d s+q a_{n-k}=0 .
$$

Assume that $x(t)=e^{\lambda t}, t \in[n, n+1)$, then from (2.4), we get

$$
f(\lambda)=e^{\lambda}-1+\frac{p e^{-\lambda t}}{\lambda}(\lambda-1)+q e^{-\lambda k}=0
$$

Putting $e^{\lambda}=\gamma$ in Eqn.(2.5),then

$$
F(\gamma)=\gamma-1+\frac{p \gamma^{-\tau}}{\ln \gamma}(\gamma-1)+q \gamma^{-k}=0 .
$$

and consequently Eqn.(2.6) has no positive real roots if and only if Eqn.(2.5) has no real roots.Assume that Eqn.(2.5) has no real roots, then $\lambda \neq 0$, and consequently $\gamma \neq 1$. If $p=0$, then Eqn. (2.6) reduces to Eqn.(1.8),also if $q=0$,then Eqn.(2.6) reduces to Eqn.(1.5) .

THEOREM 2.2. Equation (1.1) is oscillatory if and only if its characteristic equation (2.6) has no positive real roots.

PROOF: Assume that the characteristic equation (2.6) has a positive real root $\gamma_{0}$, then $\gamma_{0}^{\prime}$ is a solution of Eqn.(2.4) which is a nonoscillatory solution and consequently Eqn.(1.1) is not oscillatory .On the other hand , assume that $x(t)>0 \forall t \in[n, n+1)$ for sufficiently large $n$ and $F(\gamma)$ has no positive real roots .As $F(\infty)=\infty$, it follows that $F(\gamma)>0 \forall \gamma \in(0, \infty)$. For seeking the contradiction, choose :

(i) $p \leq 0$ and $q \leq 0$ then $F(\gamma)<0 \forall \gamma \in(0,1)$,

(ii) $p \geq 0, q<0$ with $p<|q|$ and $\tau \leq k$ then $F(\gamma)<0 \forall \gamma \in(0,1)$,

(iii) $p<0, q \geq 0$ with $q<|p|(1-1 / \mathrm{e})$ and $\tau=k$ then $F(1 / \mathrm{e})<0$,

(iv) $p \geq 0, q \geq 0$ with $p+q \leq 1 / 8 e^{k}$ and $\tau \leq k$ then $F(1 / e)<0$, which is a contradiction.

THEOREM 2.3. If $p, q \in \Re^{+}$, then all solutions of equation (1.1) are oscillatory if and only if

$$
p e \tau+q \frac{(k+1)^{k+1}}{k^{k}}>1, k \geq 1
$$

PROOF: Assume that Eqn.(1.1) has a nonoscillatory solution, then the characteristic Eqn.(2.6) has a positive real root $\gamma_{0} \in(0,1)$. Otherwise $F\left(\gamma_{0}\right)>0 \forall \gamma_{0} \in[1, \infty)$ and therefore, we have 


$$
F\left(\gamma_{0}\right)=\gamma_{0}-1+\frac{p \gamma_{0}^{-\tau}}{\ln \gamma_{0}}\left(\gamma_{0}-1\right)+q \gamma_{0}^{-k}=0, \gamma_{0} \in(0,1)
$$

and then

$$
\begin{aligned}
0 & =\left(\gamma_{0}-1\right)\left\{1+\frac{p \gamma_{0}{ }^{-\tau}}{\ln \gamma_{0}}+q \gamma_{0}^{-k} /\left(\gamma_{0}-1\right)\right\} \\
0 & =1+\frac{p \gamma_{0}{ }^{-\tau}}{\ln \gamma_{0}}+q \gamma_{0}^{-k} /\left(\gamma_{0}-1\right) \\
& \leq 1-p e \tau-q \frac{(k+1)^{k+1}}{k^{k}}
\end{aligned}
$$

which is a contradiction .On the other hand ,assume that

$$
\text { pe } \tau+q \frac{(k+1)^{k+1}}{k^{k}} \leq 1, k \geq 1 .
$$

Now, we study the following cases:

(1) $q=0, \mathrm{p}>0$.

Since $F(\gamma)>0, \forall \gamma \in(1, \infty)$ and $F\left(e^{-\frac{1}{r}}\right) \leq 0$, then there exists $\gamma_{1} \in \mathfrak{R}^{+}$such that $F\left(\gamma_{1}\right)=0$. i.e. the characteristic equation has a positive real root and consequently equation (1.1) is not oscillatory.

(2) $p=0, q>0$.

In this case, $F(\gamma)>0, \forall \gamma \in(1, \infty)$ and $F\left(\frac{k}{k+1}\right) \leq 0$.Therefore, the characteristic equation has a positive real root and then equation(1.1) has a nonoscillatory solution.

(3) $p>0, q>0$.

Since $p e \tau+q \frac{(k+1)^{k+1}}{k^{k}} \leq 1$,

then,

$$
q \frac{(k+1)^{k+1}}{k^{k}}+\frac{p \frac{(k+1)^{\tau+1}}{k^{\tau}}}{\ln \left(\frac{k}{k+1}\right)}<p e \tau+q \frac{(k+1)^{k+1}}{k^{k}} \leq 1, k \geq 1 .
$$

It is clear that the characteristic equation has no real roots in $(1, \infty)$ and $F(\gamma)>0$, but

$$
\begin{aligned}
F\left(\frac{k}{k+1}\right) & =\frac{k}{k+1}-1+\frac{p \frac{(k+1)^{\tau}}{k^{\tau}}}{\ln \left(\frac{k}{k+1}\right)}+q \frac{(k+1)^{k}}{k^{k}} \\
& =-\frac{1}{k+1}+\frac{q}{(k+1)} \frac{(k+1)^{k+1}}{k^{k}}+\frac{p}{k+1} \frac{\frac{(k+1)^{\tau+1}}{k^{\tau}}}{\ln \left(\frac{k}{k+1}\right)} \text { From (2.8), it follows that } F\left(\frac{k}{k+1}\right) \leq 0 \text { and }
\end{aligned}
$$

consequently equation (1.1) has a nonoscillatory solution.

REMARK. If $\tau=k=1$ and $p, q \in \mathfrak{R}^{+}$, then $p e+4 q>1$ is a necessary and sufficient condition for oscillation of

$$
\dot{x}(t)+p x(t-1)+q x([t-1])=0 .
$$




\section{REFERENCES}

[1] COOKE, K.L. and WIENER,J., Retarded differential equations with piecewise constant delays , $J$. Math. Anal. and Appl. 99 (1984), 265- 294.

[2] COOKE, K.L. and WIENER,J., Neutral differential equations with piecewise constant argument, Bolletino Unione Mathematica Italiana 1-B (1987), 321-345.

[3] GROVE, E.A. , GYÖRI,I.,and LADAS,G.,On the characteristic equations for equations with continuous and piecewise constant arguments, Radovi Mathematicki 5 (1990), 271- 281.

[4] GYÖRI,I.,and LADAS,G. , Oscillation Theory of Delay Differential Equations with Applications , Clarendon Press, Oxford ,1991.

[5] WIENER,J. and COOKE, K.L., Oscillations in systems of differential equations with piecewise constant argument, J. Math. Anal. and Appl. 137 (1989), 221-239 .

[6] WIENER, J., Generalized Solutions of Functional Differential Equations , World Scientific , Singapore, 1993. 


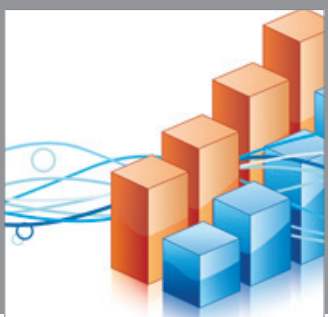

Advances in

Operations Research

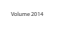

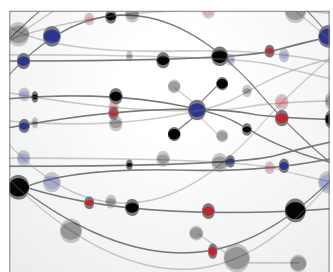

\section{The Scientific} World Journal
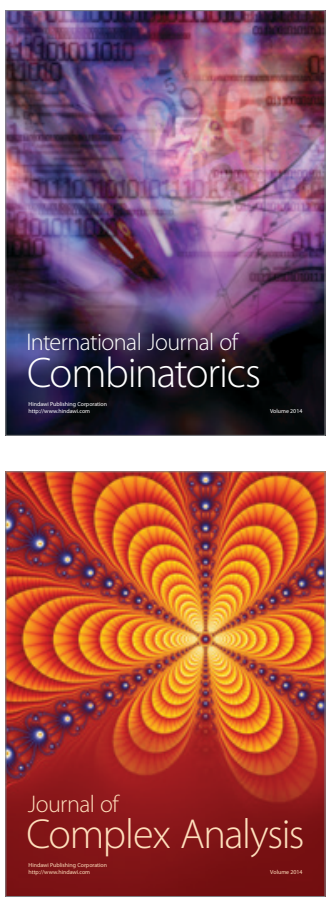

International Journal of

Mathematics and

Mathematical

Sciences
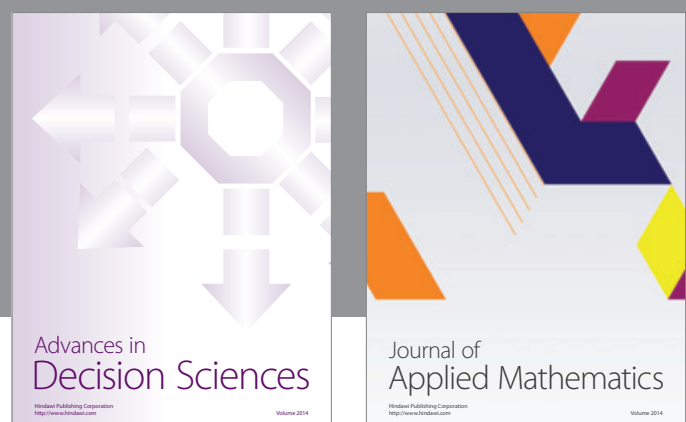

Journal of

Applied Mathematics
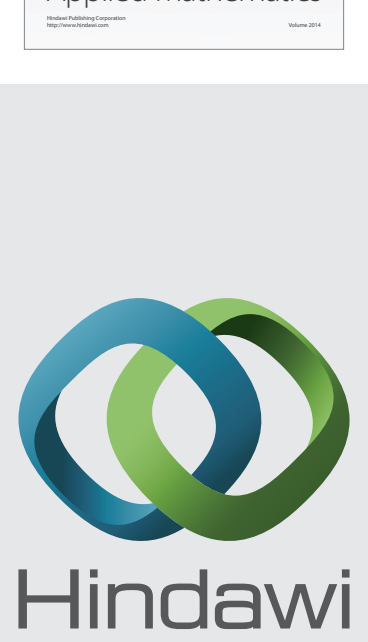

Submit your manuscripts at http://www.hindawi.com
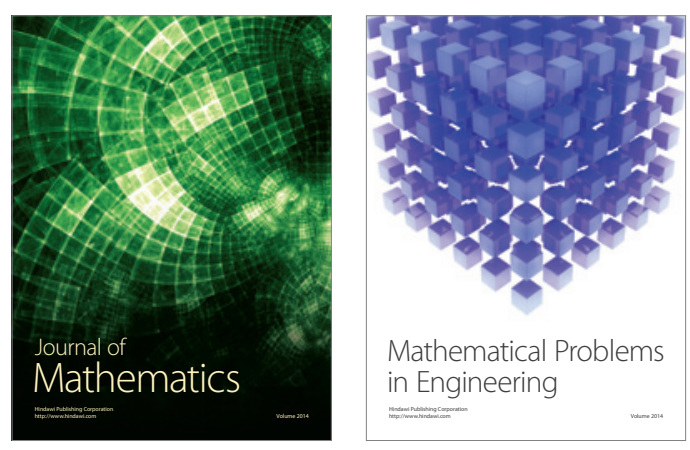

Mathematical Problems in Engineering
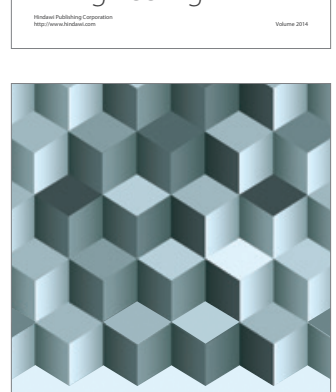

Journal of

Function Spaces
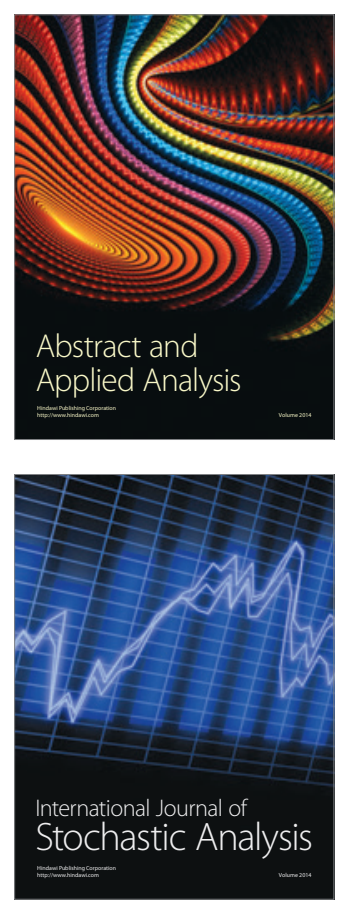

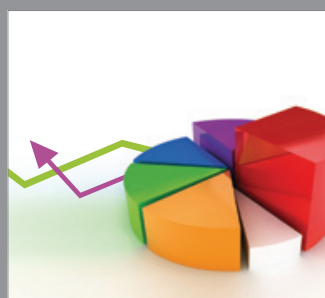

ournal of

Probability and Statistics

Promensencen
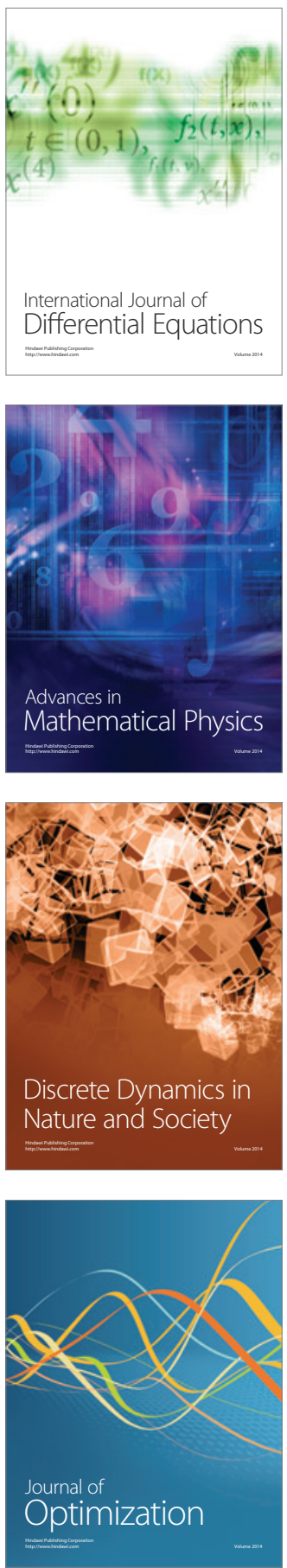